\title{
Produção acadêmica de autores brasileiros sobre Internacionalização: Balanço das publicações no Brasil no Séc. XXI
}

\author{
Sergio Garrido Moraes ${ }^{1}$, Vivian lara Strehlau e Frederico Araújo Turolla \\ Escola Superior de Propaganda e Marketing - ESPM, São Paulo/SP, Brasil
}

\section{DETALHES DO ARTIGO}

\section{Histórico do artigo:}

Recebido em 31 de Outubro de 2014

Aceito em 17 de Julho de 2015

Disponível online em 30 de Agosto de 2015

Sistema de Revisão "Double Blind Review"

Editores científicos

Gabriel Vouga Chueke

Marcos Amatucci

\section{Palavras-chaves:}

Internacionalização

Produção acadêmica

Balanço da literatura

\section{RESUMO}

Este artigo apresenta uma análise da pesquisa científica brasileira em Internacionalização de Empresas, mediante a revisão de todos os artigos publicados na área nos periódicos classificados como Qualis A pela CAPES, no período de 2001 a 2014 e sua comparação com alguns indicadores de outros estudos realizados sobre a pesquisa acadêmica no exterior e no Brasil, tanto em internacionalização quanto em temas da administração em geral. A análise se concentra na temática abordada, na metodologia empregada, nas teorias sobre internacionalização discutidas e na demografia autoral. Constatou-se que há possíveis diferenças na pesquisa sobre internacionalização brasileira em comparação à que se pratica em outros países em relação a temática, metodologia e teorias. No âmbito da demografia autoral, aponta-se que a contribuição das IES públicas e privadas encontra-se em linha com outras áreas. Sugerem-se, ao final, possibilidades de ampliação do estudo para melhor refletir a produção científica brasileira em Internacionalização de Empresas.

(C) 2015 Internext | ESPM. Todos os direitos reservados!

\section{Introdução}

A expansão dos cursos de pós-graduação em administração no Brasil e o exercício de maior pressão pela Coordenadoria de Aperfeiçoamento do Ensino Superior (CAPES) foram fatores de estímulo à produção acadêmica e sua divulgação sistemática em periódicos de excelência e abrangência nacional (JABBOUR; SANTOS, BARBIERI, 2008). Esse incremento levou ao surgimento de vários balanços dessa produção científica, visando a sintetizar os avanços da pesquisa acadêmica na área de gestão. Esses balanços foram realizados na área de estudos organizacionais, marketing, estratégia, finanças, gestão de operações, gestão ambiental, gestão de recursos humanos, empreendedorismo, terceiro setor, e sistemas de informação (OGASAVARA; MASIERO, 2009).

\footnotetext{
${ }^{1}$ Contato do autor: Email: smoraes@espm.br
}

Os balanços da produção científica na área de administração começaram a ser produzidos e divulgados antes da década de 90, cabendo o pioneirismo às pesquisas de Siqueira (1988) e de Machado-da-Silva, Cunha e Amboni (1990), realizadas nas áreas de recursos humanos e organizações, respectivamente, e divulgados no EnANPAD (Encontro da Associação Nacional de PósGraduação em Administração) (JABBOUR; SANTOS; BARBIERI, 2008), sem grande destaque nos periódicos de maior prestígio. No entanto, em 2003 a RAE (Revista de Administração de Empresas da EAESP-FGV) publicou um dossiê com quatro balanços da pesquisa acadêmica de quatro áreas distintas: marketing (VIEIRA, 2003), recursos humanos (TONELLI; CALDAS; LACOMBE; TINOCO, 2003), finanças (LEAL; OLIVEIRA; SOLURI, 2003) e operações (ARKADER, 2003). O editorial da revista apresenta os estudos como trazendo o estado da arte da pesquisa

(C) 2015 Internext | ESPM. Todos os direitos reservados! Doi: dx.doi.org/10.18568/1980-486510282-962015 
acadêmica nessas áreas no Brasil. A partir daí, praticamente todas as áreas da administração foram mapeadas em artigos na revista, todos enquadrados na rubrica RAE-Documento. Ainda em 2003 foram publicados mais três balanços da produção acadêmica brasileira: estudos críticos da administração (ALCADIPANI; DAVEL, 2003), estratégia empresarial (BERTERO; VASCONCELOS; BINDER, 2003), e administração pública (PACHECO, 2003). Em 2004 mais um meta-estudo foi publicado, novamente sobre recursos humanos (CALDAS; TINOCO, 2004). Em 2005 é a vez da área de sistemas de informação ser contemplada com uma revisão da produção científica (HOPPEN; MEIRELLES, 2005), além de contabilidade (CARDOSO; MENDONÇA NETO; RICCIO; SAKATA, 2005), que aparece novamente em 2009 (MENDONÇA NETO; CARDOSO; RICCIO; SAKATA, 2009). A esses estudos juntam-se o publicado na RAC em 2008 por Jabbour, Santos e Barbieri, sobre gestão ambiental, e o apresentado no EnANPAD de 2009 por Ogasavara e Masiero, sobre internacionalização de multinacionais brasileiras. A produção mais recente de estudos sobre a publicação em administração contempla as áreas tradicionais, como marketing (ANDRADE; JOAQUIM, GOSLING, 2012), mas também dá ênfase a recortes mais específicos, como RBV (FAVORETO; AMÂNCIO-
VIEIRA, SHIMADA, 2014), qualidade em serviços (PEREIRA; CARVALHO, ROTONDARO, 2013), além da análise dos periódicos (ARAÚJO; AZEVEDO, VIEIRA, NASCIMENTO, 2014). Uma relação não exaustiva de artigos com estudos e bibliometrias sobre a área de administração encontra-se na tabela 1, com os temas e parâmetros abordados.

Os parâmetros mais comuns de análise nesses meta-estudos são a temática abordada e a metodologia empregada. Também a autoria (número de autores e/ou nome) e sua filiação a instituição acadêmica são estudadas. Alguns trabalhos fazem levantamento das citações, quanto a nacionalidade dos autores, qualidade das fontes, etc. Poucos trabalhos se dedicam a levantar e analisar a teoria empregada nos artigos selecionados.

As fontes do material analisado são, em sua maioria, os periódicos Revista de Administração de Empresas (RAE), da EAESP/FGV, Revista de Administração da USP (RAUSP), e Revista de Administração Contemporânea (RAC), nem sempre com justificativa da escolha. Também outras revistas são utilizadas como fonte, sempre com justificativa pela avaliação recebida pelo Qualis da CAPES (revistas classificação A). Alguns estudos analisam, ainda, trabalhos apresentados no EnANPAD, em conjunto

Tab. 1

Artigos com balanços da produção acadêmica no Brasil

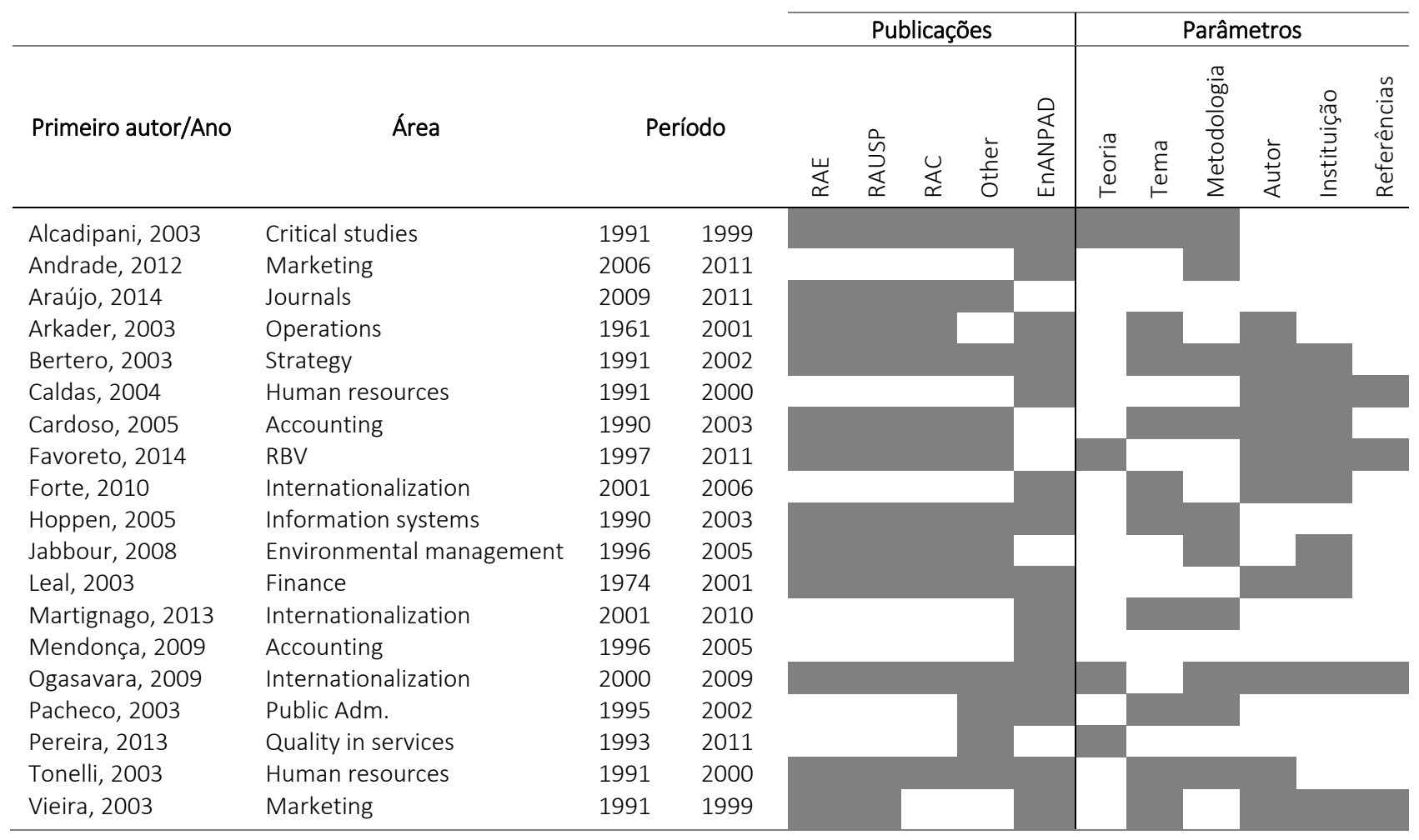

Fonte: Artigos citados, organização dos autores 
com as revistas ou não. O período de tempo mais comumente analisado é de dez anos, seja como década, seja como período de tempo quebrado.

Praticamente todas as áreas da administração foram cobertas nos últimos anos com balanços sobre a produção acadêmica relativa a elas, notadamente em artigos publicados na RAE, conforme se vê na tabela 1. A área de internacionalização de empresas aparece com três artigos: (a) Ogasavara e Masiero (2009), apresentado no EnANPAD, que analisa os artigos que tratam da internacionalização de empresas brasileiras, como Ambev, JBS-Friboi, e Vale; os autores estudaram o campo por meio de estudo bibliométrico, mas se limitado aos estudos que definiram a internacionalização como um processo desenvolvido por empresas que realizam Investimento Externo Direto (IED); (b) Forte, Rodrigues e Oliveira (2010), que teve por objetivo descrever a evolução das pesquisas científicas em gestão internacional na academia brasileira, analisando os trabalhos apresentados no EnANPAD de 2001 a 2006, mediante o levantamento, para cada trabalho, de autoria, instituição, estado e temática; os autores finalizam sugerindo, entre outras ações, que a futura pesquisa sobre o assunto inclua uma avaliação da metodologia de pesquisa empregada pelos trabalhos, e que seja ampliada para os papers dos periódicos Qualis A e B; e (c) Martignago, Alperstedt e Cário (2013), que mapearam a pesquisa
Análises bibliométricas, por sua vez, contribuem para aprofundar a compreensão sobre a qualidade da produção acadêmica de uma área do conhecimento, pois possibilitam extrair informações para avaliar o comportamento das pesquisas científicas na área (CAMPOS, 2003). Com o surgimento dos programas de pós-graduação em gestão internacional (ESPM e EAESP/FGV, entre outros), falta à área um estudo que retrate a sua produção de alto nível publicada nos periódicos mais bem avaliados.

Assim, para atender a essa demanda e procurar ampliar a pesquisa sobre os estudos acadêmicos brasileiros relativos à internacionalização, o presente trabalho aborda a seguinte problemática de pesquisa: quais as principais características da produção acadêmica brasileira em gestão internacional, em termos de metodologia, temática, teorias e demografia autoral, registrada em periódicos da área de administração Qualis A entre 2001 e 2014?

\section{Metodologia}

A produção acadêmica em internacionalização de empresas abordada nesse trabalho constitui-se de trabalhos publicados nos periódicos da área de Administração, Contabilidade de Turismo, da CAPES (CAPES, 2014), classificados no grupo A, o que totaliza 56 periódicos.

Tab. 2

Periódicos analisados

\begin{tabular}{|c|c|c|c|c|c|c|c|c|c|}
\hline \multirow[b]{2}{*}{ ISNN } & \multirow[b]{2}{*}{ Título } & \multicolumn{2}{|l|}{ CAPES } & \multicolumn{2}{|c|}{ SClmago } & \multicolumn{2}{|c|}{ Google Scholar } & \multicolumn{2}{|c|}{ Scielo } \\
\hline & & Qualis & SJR & $\begin{array}{c}\text { Cit. p/ } \\
\text { Doc }\end{array}$ & $\begin{array}{l}\text { Total } \\
\text { cit. }\end{array}$ & Index & Median & IF2 & IF3 \\
\hline 1807-7692 & BAR - Brazilian Administration Review & A2 & 0,17 & - & - & - & - & 0,037 & 0,052 \\
\hline $1808-2386$ & BBR - Brazilian Business Review & A2 & - & - & - & - & - & & \\
\hline 0104-530x & G\&P - Gestão \& Produção (UFSCar) & A2 & 0,16 & 0,1 & 38 & 3 & 5 & 0,104 & 0,114 \\
\hline $1984-9230$ & O\&S - Organização \& Sociedade & A2 & - & - & - & - & - & 0,108 & 0,103 \\
\hline 1982-7849 & RAC - Revista de Administração Contemporânea & A2 & - & - & - & 15 & 20 & 0,127 & 0,128 \\
\hline 0034-7590 & RAE - Revista de Administração de Empresas & A2 & 0,21 & 0,24 & 36 & 13 & 24 & 0,257 & 0,318 \\
\hline 0080-2107 & RAUSP - Revista de Administração da USP & A2 & - & - & - & 10 & 17 & 0,019 & 0,027 \\
\hline 0034-7612 & RAP - Revista de Administração Pública & A2 & 0,20 & 0,11 & 31 & 19 & 32 & 0,103 & 0,120 \\
\hline
\end{tabular}

Fonte: Desenvolvido pelos autores

em Gestão Internacional de 2001 a 2010, mas apenas nos anais do EnANPAD.

Por outro lado, Splitter, Rosa e Borba (2012) avaliaram 194 trabalhos apresentados no EnANPAD entre 2000 e 2011 e classificados como estudos bibliométricos e não registraram nenhum sobre gestão internacional e/ou internacionalização.
Foram escolhidos apenas os periódicos com classificação A em função das novas diretrizes de avaliação da CAPES, que privilegiam as publicações nesse nível, visando à melhor qualificação da produção nacional (PNPG, 2013). Em uma primeira seleção, foram excluídos os periódicos das subáreas de medicina, saúde, engenharia, enfermagem, psicologia, e outras correlatas, restando 24 
publicações. Em seguida, foram excluídas as duplicações de um mesmo periódico com ISSN para edição on line e impressa, restando 13 que foram investigados. No final, apenas oito revistas Qualis A trouxeram artigos sobre internacionalização, todos elas de classificação A2. As versões eletrônicas de algumas revistas não foram destacadas pois: RACe só teve 7 números, entre 2007 e início de 2009 e a busca no site da RAC traz os artigos da RACe; RAEe foi incorporada à RAE em 2011 e a busca no site da RAE traz os artigos da RAEe; RAUSPe só teve dois números em 2008, e nenhum traz artigos sobre internacionalização.

A escolha de periódicos e não de trabalhos registrados em anais do EnANPAD é devida ao fato de - CAPES não considerar a produção acadêmica veiculada em anais de congressos e eventos como qualificada (JABBOUR; SANTOS, BARBIERI, 2008). Além disso, os balanços críticos feitos nos últimos anos têm evitado, em geral, combinar o levantamento e análise de trabalhos publicados no EnANPAD e nos periódicos, pois o volume de trabalhos em qualquer área do EnANPAD é maior do que na soma dos periódicos em qualquer período e os critérios de aprovação para publicação nos periódicos são mais seletivos e rigorosos (TONELLI; CALDAS, LACOMBE, TINOCO, 2003).

Definidas as fontes do material a ser analisado, delimitou-se a dimensão temporal da cobertura como sendo desde o início da década / século - de 2001 a 2014. Esse período de investigação vai além dos dez anos comumente utilizados em metaestudos analisados (ALCADIPANI; DAVEL, 2003; BERTERO; VASCONCELOS; BINDER, 2003; CALDAS; TINOCO, 2004; MARTIGNAGO; ALPERSTEDT; CÁRIO, 2013; MENDONÇA NETO; CARDOSO, RICCIO, SAKATA, 2009; TONELLI; CALDAS, LACOMBE, TINOCO, 2003; VIEIRA, 2003). Segundo Teóphilo e Iudícibus (2005) um horizonte temporal de 10 anos é período suficiente para se avaliarem tendências da produção científica a ser estudada. Nesse caso, a maior duração visa contemplar desde o ano de 2001, quando foi criada a área de gestão internacional no EnANPAD, passando a ter identidade própria e não mais um tema dentro da área de estratégia, e dar o panorama completo do período de maior crescimento na publicação da área (FORTE, RODRIGUES, OLIVEIRA, 2010). No plano temático, esse período coincide com a proposta de Buckley (2002), que previa a superação dos temas vigentes à época por uma nova agenda de pesquisa.
Os artigos foram selecionados nos sites dos periódicos escolhidos. Para a seleção foram utilizados os sistemas de busca dos sites, procurando pelas palavras mais comuns que sinalizam a área de estudo, como "internacionalização", "multinacional(is)", "subsidiária(s)", "globalização", além dos nomes de alguns autores mais frequentes nos artigos sobre internacionalização, como Ângela da Rocha, Eduardo Vasconcellos, Felipe Borini, Sérgio Rezende, Fleury, entre outros citados como mais produtivos em estudos anteriores (OGASAVARA; MASIERO, 2009). Os textos foram avaliados pelo título, resumo e estrutura, para inclusão ou não no corpus da pesquisa. Alguns textos foram recusados, por abordarem assuntos que, apesar de conter palavraschave, fugiam ao escopo da pesquisa, como acessibilidade da produção científica, ou metaestudo de gestão ambiental, por exemplo.

As variáveis analisadas para esse estudo são: (a) temática; (b) metodologia; (c) demografia de autoria; e (d) teorias de internacionalização.

Tab. 3

Categorias temáticas

\begin{tabular}{|c|c|}
\hline BUCKLEY, 2002 & AMATUCCI, 2006a \\
\hline Fluxos de IDE & $\begin{array}{l}\text { INT - Internacionalização } \\
\text { Joint Ventures } \\
\text { M\&A } \\
\text { Alianças estratégicas } \\
\text { Empreendedorismo }\end{array}$ \\
\hline $\begin{array}{l}\text { Existência, estratégia e } \\
\text { organização de EMNs }\end{array}$ & $\begin{array}{l}\text { GMN - Gerenciamento da EMN } \\
\text { Governança corporativa } \\
\text { Finanças e risk management } \\
\text { Cross-cultural management } \\
\text { International branding }\end{array}$ \\
\hline $\begin{array}{l}\text { Globalização e } \\
\text { novas formas de IB }\end{array}$ & $\begin{array}{l}\text { AMB - Análise do ambiente } \\
\text { Gerenciamento de R. H. } \\
\text { Relação MNE-governo } \\
\text { Teoria e metodologia } \\
\text { Outros }\end{array}$ \\
\hline
\end{tabular}

Fonte: Amatucci (2006a)

Para classificar a temática, recorreu-se a Amatucci (2006a), em estudo que analisou 199 artigos sobre internacionalização publicados no Journal of International Business Studies (JIBS), do período de 2001 a 2006. Nesse estudo, o autor adapta e amplia a classificação sugerida por Buckley (2002), que classifica a agenda de pesquisa sobre internacionalização de acordo com a época, os tópicos de estudo e o país ou região mais enfatizado. Assim, chega a três diferentes agendas, a que chama de: (a) fluxo de Investimento Direto no Exterior (IDE), englobando pesquisas e autores do final da segunda 
guerra até os anos 70, e com foco na internacionalização das empresas americanas para a Europa, América Latina e Canadá; (b) gerenciamento de empresas multinacionais (EMNs), agenda que vai dos anos 70 até os 90, foca os países subdesenvolvidos, Japão e Tigres Asiáticos; e (c) globalização, desde meados dos anos 80 até a época da redação do artigo, 2000, aborda a Europa do Leste, a crise asiática e a China. Para efeito de classificação da temática dos artigos analisados, Amatucci (2006a) desdobra as categorias propostas por Buckley (2002), com base no que o material disponibilizava, chegando a uma lista de 17 temas, depois reagrupados em três novas categorias, finalizando com a taxonomia mostrada na tabela 3.

A identificação da temática empregada foi feita pela leitura do resumo, da seção revisão da literatura, ou, quando necessário, a introdução ou o artigo todo. extremos de contexto, generalização e precisão, chamados de "o dilema dos três chifres". Os oito tipos de estratégias de McGrath (1982) são assim divididos:

- estratégias que acontecem no próprio contexto do participante: estudos de campo e experimento de campo (têm o máximo grau de contexto)

- estratégias que acontecem em contextos irreais: simulação experimental e experimento de laboratório (máximo grau de controle e precisão);

- estratégias que acontecem independente do contexto, ou até anulando-o: surveys e tarefas de julgamento (alto grau de generalização);

- estratégias que acontecem sem participantes, em que não há comportamento: teoria formal e simulação computadorizada.

Tab. 4

Metodologias de pesquisa

\begin{tabular}{cl}
\hline SIGLA & METODOLOGIA \\
\hline TEO & $\begin{array}{l}\text { Teoria formal / revisão de literatura: desenvolvimentos com base em ideias sem relação com pesquisas empíricas } \\
\text { imediatas; envolve processo indutivo; } \\
\text { Survey: pesquisas quantitativas, cujos dados são oriundos de questionários preenchidos pelos respondentes, com ou } \\
\text { sem ajuda do pesquisador; contexto é neutralizado; }\end{array}$ \\
LAB & $\begin{array}{l}\text { Experimento de laboratório: traz os participantes para um arranjo artificial, especialmente organizado para os } \\
\text { propósitos da pesquisa; maior precisão na medição de comportamento; }\end{array}$ \\
SEX & $\begin{array}{l}\text { Simulação experimental: pesquisadores recriam o contexto do fenômeno que querem estudar; apresenta precisão } \\
\text { moderadamente alta, realismo de contexto, mas baixa generalização; }\end{array}$ \\
EC1 & $\begin{array}{l}\text { Estudo de campo primário (dados primários): os pesquisadores vão até o local fazer a coleta de dados; máximo } \\
\text { realismo de contexto, mas baixas generalização e precisão; }\end{array}$ \\
EC2 & $\begin{array}{l}\text { Estudo de campo secundário (dados secundários): os pesquisadores utilizam dados coletados previamente, por outro } \\
\text { pesquisador, uma agência ou órgão governamental; }\end{array}$ \\
EXC & $\begin{array}{l}\text { Experimento de campo: os pesquisadores vão até o fenômeno e realizam uma simulação com os participantes no } \\
\text { próprio local de trabalho; baixa generalização; }\end{array}$ \\
JUL & $\begin{array}{l}\text { Tarefas de julgamento: os participantes são levados a classificar objetos trazidos pelos pesquisadores; amostras } \\
\text { geralmente não representativas; }\end{array}$ \\
SCO & $\begin{array}{l}\text { Simulação computadorizada: os pesquisadores criam um modelo de fenômeno e estudam a interação entre as } \\
\text { variáveis; baixa precisão, alta generalização e realismo; } \\
\text { Survey com técnicas qualitativas; }\end{array}$ \\
S\&Q & $\begin{array}{l}\text { Estudo de campo secundário com técnicas qualitativas. } \\
\text { ECQ }\end{array}$
\end{tabular}

Fonte: Amatucci (2006b), Scandura and Williams (2000), McGrath (1982)

Para a classificação da variável metodologia, foram utilizados os critérios adaptados por Amatucci (2006b) da obra de Scandura e Williams (2000), que, por sua vez, basearam-se no trabalho de McGrath (1982), que define oito estratégias de pesquisa. Essas estratégias são classificadas de acordo com a particularidade ou universalidade do comportamento, e com o grau de intrusão da pesquisa, além de uma terceira dimensão: os
A essas oito estratégias puras de McGrath, Scandura e Williams (2000) acrescentam mais uma, dividindo os estudos de campo entre os que utilizam dados primários e os que utilizam dados secundários; além disso, incluem na estratégia "teoria formal" a "revisão de literatura". Amatucci (2006b) acrescenta, ainda, mais dois tipos, que buscam captar a tendência contemporânea de combinar, no mesmo estudo, estratégias quantitativas com qualitativas. 0 
resultado são onze estratégias diferentes de pesquisa, conforme listado na tabela 4.

A identificação da metodologia empregada foi feita pela leitura do resumo, da seção de metodologia, ou, quando necessário, a introdução ou o artigo todo.

Para a variável demografia de autoria alguns estudos utilizam o critério de adotar a proporcionalidade para a autoria dos artigos (TONELLI; CALDAS, LACOMBE, TINOCO, 2003). No entanto, como a CAPES contabiliza a pontuação plena para cada autor de artigo, optou-se por atribuir um ponto a cada autor e a cada instituição de ensino superior (IES). A identificação dos autores e instituições encontrava-se na primeira página dos artigos, com exceção dos exemplares mais antigos da RAC, que trazia a qualificação dos autores em área à parte.

\subsection{Revisão teórica}

O interesse pelo tema da internacionalização é relativamente recente nas pesquisas da área de administração. Até os anos 90, os negócios internacionais eram objeto de trabalhos da sociologia, da economia e da ciência política, quase sempre a partir do paradigma da "teoria da dependência" (BERTERO, 2006). Ainda segundo Bertero (2006, slides 4 a 6),

A explicação para a emergência de uma área de interesse específico por gestão internacional deve-se aos seguintes fatores:

- Número crescente de empresas brasileiras envolvidas com negócios internacionais de diversas maneiras.

- Aumento da importância das subsidiárias brasileiras de empresas multinacionais.

- O impacto crescente, a partir da década de oitenta, do movimento de fusões de aquisições, onde EMNs são particularmente ativas como compradoras e como vendedoras.

- Gradual superação de nosso provincianismo, historicamente construído, mas que começa a diminuir. (Slides 4 a 6 )

A base teórica dos estudos sobre internacionalização é eclética e etnocêntrica, notadamente nos estudos realizados nos Estados Unidos (BERTERO, 2006). Forte, Rodrigues e Oliveira (2010) sugerem utilizar

Tab. 5

Teorias de internacionalização

\begin{tabular}{|c|c|c|c|c|c|c|}
\hline & THEORIES & $\begin{array}{l}\text { BORINI } \\
\text { et al. (2004) }\end{array}$ & $\begin{array}{c}\text { HEMAL; } \\
\text { HELAIS (2004) }\end{array}$ & $\begin{array}{l}\text { FORTE et al. } \\
\qquad(2010)\end{array}$ & DIB; CARNEIRO (2007) & MARIOTTO (2007) \\
\hline \multirow{8}{*}{ 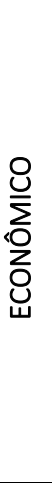 } & Internalização & Buckley; & Buckley; & Buckley; & Buckley; & Buckley; Casson (1976); \\
\hline & & Rugman (1980) & & & Teece $(1981,1986)$ & Hennart (2001) \\
\hline & Custos de Transação & Williamson (1975) & & & $\begin{array}{l}\text { Coase (1937), } \\
\text { Williamson }(1975,1979)\end{array}$ & $\begin{array}{l}\text { Coase (1937); } \\
\text { Williamson (1975) }\end{array}$ \\
\hline & Paradigma Eclético & $\begin{array}{l}\text { Dunning } \\
(1981,1993)\end{array}$ & Dunning (1980) & Dunning & $\begin{array}{l}\text { Dunning } \\
(1977,1980 \text { and 1988) }\end{array}$ & Dunning $(1977,1988)$ \\
\hline & $\begin{array}{l}\text { Poder de Mercado / } \\
\text { Organização Industrial* }\end{array}$ & $\begin{array}{l}\text { Hymer }(1960) \\
\text { Caves }(1971)^{*}\end{array}$ & $\begin{array}{l}\text { Hymer } \\
(1960,1970)\end{array}$ & Hymer & Hymer $(1960,1976)$ & Hymer (1960) Caves (1971) \\
\hline & Ciclo do Produto & Vernon (1966) & R. Vernon (1966) & Vernon & & Vernon (1966) \\
\hline & Vantagem Competitiva & Porter (1990) & & & & Krugman (1991) Porter (1990) \\
\hline & RBV & & & & & Barney (1991) \\
\hline \multirow{3}{*}{ 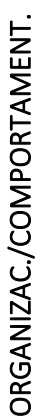 } & $\begin{array}{r}\text { Escola*/Modelo** de } \\
\text { Uppsala }\end{array}$ & $\begin{array}{l}\text { Johanson; Valhne } \\
(1977,1990)^{*}\end{array}$ & $\begin{array}{l}\text { Johansson; } \\
\text { Vahlne (1977)* }\end{array}$ & $\begin{array}{l}\text { Johansson; } \\
\text { Vahlne* }\end{array}$ & $\begin{array}{l}\text { Johanson; Wiedersheim } \\
\text { (1975) Johanson; Vahlne } \\
(1977)^{* *}\end{array}$ & Johanson; Vahlne (1977)** \\
\hline & Networks* / Redes** & $\begin{array}{l}\text { Anderson; } \\
\text { Forgren; Holm } \\
\text { (2000)* Johanson; } \\
\text { Mattson (1986)** }\end{array}$ & & & $\begin{array}{l}\text { Johanson; Mattson } \\
(1986)^{* *} \text { Forgren (1989) } \\
\text { Johanson; Vahlne } \\
(2003)^{*}\end{array}$ & $\begin{array}{l}\text { Johanson; Mattson (1986)** } \\
\text { Johanson; Vahlne }(2006)^{*}\end{array}$ \\
\hline & $\begin{array}{r}\text { Escola Nórdica* / } \\
\text { Empreendedorismo } \\
\text { internacional** / } \\
\text { Subsidiárias*** }\end{array}$ & $\begin{array}{l}\text { Birkinshaw; Hood } \\
(1998)^{* * *}\end{array}$ & $\begin{array}{l}\text { Andersson } \\
(2000)^{*}\end{array}$ & Andersson* & $\begin{array}{l}\text { Andersson }(2000)^{*} \\
\text { Birkinshaw }(1997)^{* *}\end{array}$ & \\
\hline
\end{tabular}

Fonte: Amatucci (2006b), Scandura and Williams (2000), McGrath (1982) 
referencial teórico das áreas temáticas de Estratégia nas Organizações e de Empreendedorismo da Divisão de Estratégia da ANPAD (VBR, Capacidades Dinâmicas, Processos de Mercado, Hipercompetição). No entanto, em 40 anos de estudos de Negócios Internacionais, transcorridos desde o trabalho de Hymer, algumas teorias próprias se consolidaram e são, hoje, a base desse ramo de estudos (HEMAIS; HILAL, 2004).

As teorias sobre o processo de internacionalização podem ser divididas em duas grandes áreas, representando enfoques bem diversos: o econômico e o organizacional (HEMAIS; HILAL, 2004). Vários autores se ocuparam em organizar e listar as teorias com seus principais teóricos. Borini, Coelho, Ribeiro e Proença (2004) propõem o Prisma da Internacionalização, no qual buscam a interação entre as várias teorias e modelos com o objetivo de explicar o processo de internacionalização da empresa. Hemais e Hilal (2004) resumem as principais teorias de internacionalização da empresa em uma tabela, no qual procuram mostrar que "as e variabilidade dos processos de internacionalização das empresas. Mariotto (2007) descreve as diferentes teorias com maior ênfase no pioneiro Hymer e naquelas de foco econômico. A tabela 5 resume a taxonomia das várias teorias de internacionalização e as obras consideradas pelos autores para as suas análises.

A essas abordagens já consagradas podem ser acrescentadas as mais recentes Visão Baseada em Instituições, cujo principal autor é Mike Peng, e a Global Value Chain, principalmente na obra de Gary Gereffi.

A identificação da teoria abordada foi feita pela leitura das referências, somente em relação às obras sobre internacionalização - não foram consideradas obras sobre metodologia e outras áreas de estudo. Para operacionalizar a identificação das teorias em cada artigo analisado foi selecionada uma ou, no máximo, duas das obras consideradas fundamentais pelos autores citados no quadro 5. o quadro 6 apresenta essa categorização:

Tab. 6

Obras utilizadas para identificar teorias

\begin{tabular}{ll}
\hline Teorias & Obra(s)-chave(s) \\
\hline Internalização & Buckley; Casson (1976, 1997) \\
Custos de Transação & Coase (1937) Williamson (1975) \\
Paradigma Eclético & Dunning (1977, 1980, 1981, 1988, 1993) \\
Poder de Mercado / Organização Industrial* & Hymer (1960,1970, 1976), Caves (1971)* \\
Ciclo do Produto & Vernon (1966) \\
Vantagem Competitiva & Porter (1990) \\
Visão Baseada em Recursos & Barney (1991) \\
Escola* / Modelo de Uppsala & Johanson; Valhne (1977, 1990)* \\
Networks* / Redes** & Anderson; Forgren; Holm (2000*, Johanson; Mattson (1986)** \\
Escola Nórdica* / Empreendedorismo internacional** / & Andersson (2000), Birkinshaw (1997)**, Birkinshaw; Hood (1998)*** \\
Subsidiárias*** & \\
Visão Baseada em Instituições & Mike Peng (2002, 2003, 2006, 2009) \\
Global Value Chains & Gereffi \\
\hline
\end{tabular}

Fonte: Elaborado pelos autores.

teorias são muito mais complementares do que oponentes". Carneiro e Dib (2007) abordam seis teorias consagradas sobre internacionalização e sua contribuição para responder cinco questões básicas: por que, o quê, quando, onde e como as empresas internacionalizam; apontam as limitações descritivas e explanatórias das teorias consideradas em relação às questões analisadas; e terminam sugerindo que uma nova teoria geral ou teorias específicas seriam necessárias para lidar com a crescente complexidade
A categorização de teoria, metodologia e taxonomia de internacionalização foi feita por dois dos autores; quando houve divergência, os juízes discutiram até chegar ao consenso.

\section{Resultados}

\subsection{Descrição da Amostra}

A busca nos sites dos periódicos resultou em 80 artigos selecionados, com as características (palavras 
e autores) escolhidas para o estudo. Esses 80 artigos foram produzidos por 127 autores diferentes, pertencentes a 51 instituições de ensino e pesquisa (IES).

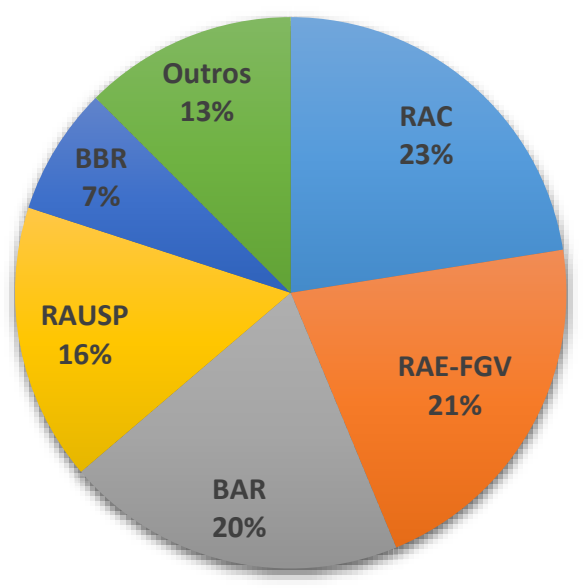

Figura 1: Artigos por revista (*Outros: GP, OS, RAP) Fonte: Elaborado pelos autores

O trabalho colaborativo foi o mais comum: apenas 14 artigos tiveram um único autor; o mais comum foram artigos escritos por duplas (38 artigos); com três autores foram 19 artigos, e nove foram escritos por quatro ou mais autores. Não foi possível detectar pelos artigos a relação entre os autores, se orientador/ orientandos em programas de pósgraduação, grupo de pesquisa ou outra.
A figura 1 mostra que as revistas mais ativas na publicação sobre internacionalização foram RAC e a RAEFGV, com quase metade (44\%) dos artigos do período.

A figura 2 demonstra certa tendência de crescimento na publicação de artigos sobre internacionalização. Nos cinco primeiros anos do período analisado, a média anual pouco passava de dois artigos $(2,4)$; nos últimos nove anos essa média passa de sete $(7,6)$ artigos. Esse incremento pode ser atribuído ao início de publicações sobre internacionalização na RAE/FGV e na BAR. O ano com maior número de artigos foi 2010, com 13 publicações.

\subsection{Temática}

As áreas temáticas que predominam na produção acadêmica brasileira são INT e GMN com $41 \%$ cada (Figura 3). A primeira é aquela classificada por Buckley (2002) como Fluxo de IDE e por Amatucci (2006a) como Internacionalização. Na taxonomia de Buckley esse tópico de estudos seria aquele que explica, principalmente, o fluxo de investimento no pós-guerra dos EUA para a Europa, e foi dominante até os anos 70. Já a área GMN explica a estratégia e a organização das empresas multinacionais estrangeiras e foi predominante de 1970 a 1990. A

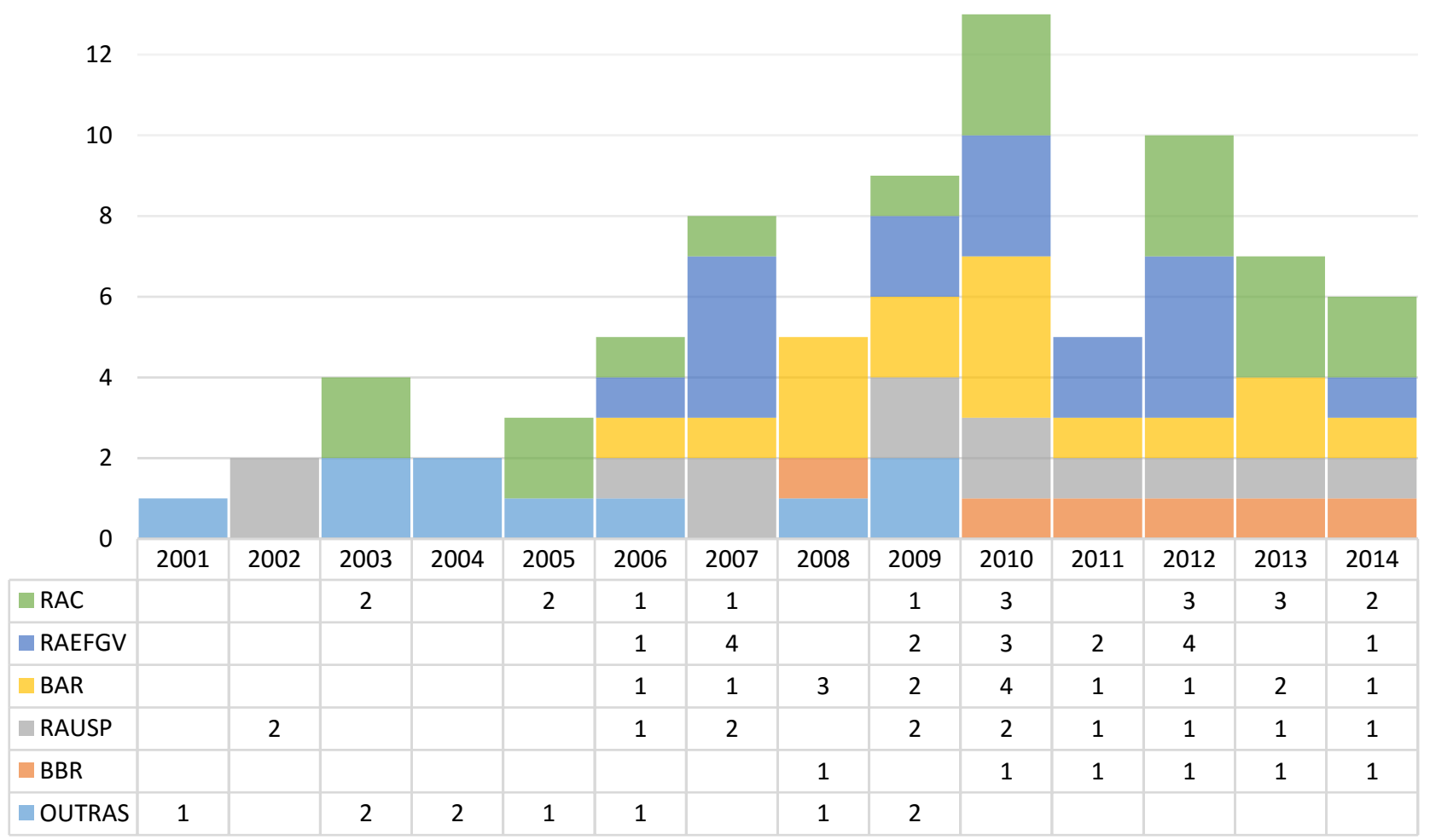

Figura 2: Produção anual

Fonte: Elaborado pelos autores 
área temática menos estudada (AMB 18\%) no Brasil considerada por Buckley (2002) a categoria mais atual à época que escreveu seu artigo - trata de jointventures, significados da globalização, competitividade, etc., e, mesmo assim, já estaria pronta para ser superada por uma nova agenda de pesquisas.

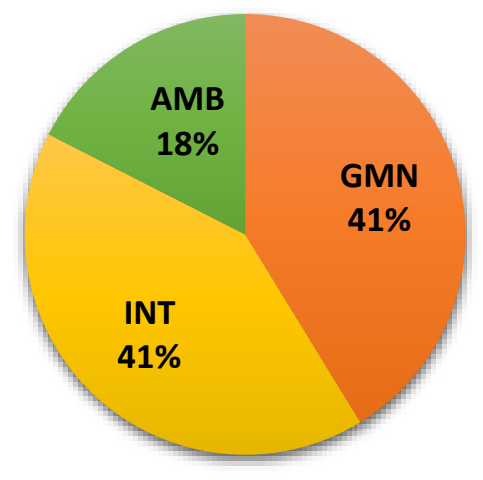

Figura 3: Categorias temáticas (INT: Internacionalização; GMN: Gerenciamento de multinacionais; AMB: Análise do ambiente) Fonte: Elaborado pelos autores

No trabalho de Amatucci (2006a) com os artigos do JIBS, a distribuição das áreas temáticas é substancialmente diferente: INT 29\%; GMN 42\%; AMB 29\%.

\subsection{Metodologia}

Quase a metade da pesquisa acadêmica brasileira sobre internacionalização (46\%) utiliza a metodologia do estudo de caso primário (figura 4). Esse resultado confirma a suspeita em Forte, Rodrigues e Oliveira (2010), de que a maioria dos trabalhos utilizaria essa metodologia (junto com "estudos exploratórios descritivos"). No âmbito internacional, Amatucci (2006a) identificou uma maior participação dos surveys nos 199 artigos que pesquisou no JIBS com $35 \%$ dos trabalhos, contra 22\% no Brasil.

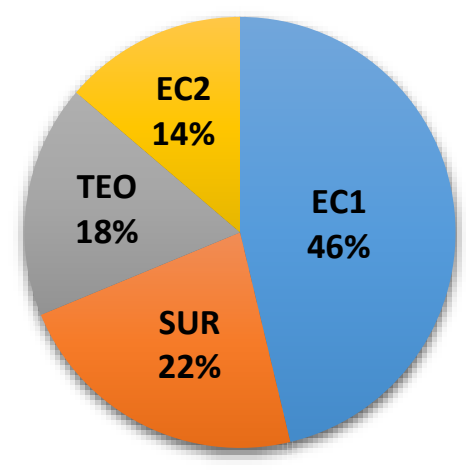

Figura 4: Metodologia (EC1: Estudo de caso/dados primários; SUR: Survey; TEO: Teoria formal/revisão de literatura; EC2: Estudo de dados secundários) Fonte: Elaborado pelos autores
O levantamento de Scandura e Williams (2000) realizado com 385 artigos publicados em três dos mais prestigiados journals americanos no período 1995-97, no entanto, revelou uma importância bem menor dos surveys (4\%), e maior para os estudos de campo (68\%), maior até do que na pesquisa brasileira.

\subsection{Teorias}

As teorias mais empregadas para a explicação dos processos de internacionalização dentro da produção acadêmica brasileira apresentam uma divisão equilibrada entre aquelas classificadas como comportamentais e as econômicas, com ligeiro predomínio das primeiras: Uppsala, Empreendedorismo e Redes. A figura 5 mostra cada uma das teorias isoladamente - se considerarmos como grupo, as comportamentais representam $46 \%$ dos trabalhos analisados (figura 6). Os estudos com base em teorias econômicas correspondem a $44 \%$ do total e $10 \%$ não tem vinculação com nenhum desses dois grandes grupos.

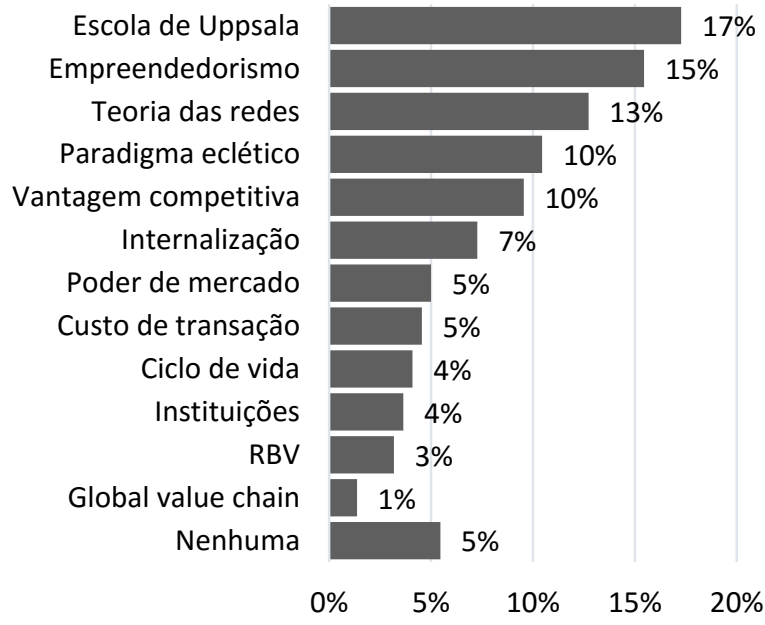

Figura 5: Referencial teórico sobre Internacionalização - \% número de citações

Fonte: Elaborado pelos autores

Na academia internacional, no entanto, os estudos de base teórica econômica (46\%) são bem mais frequentes do que os de base comportamental (25\%) (Amatucci, 2006a).

Além disso, Mariotto (2007) cita à página 69 de seu livro o artigo de 1991 de Barney sobre VBR - visão baseada em recursos ("Firm resources and sustained competitive advantage - Journal of Management) como sendo um dos mais citados de toda a literatura de negócios. Ainda de acordo com Mariotto (2007), o 
extenso uso da VBR em negócios internacionais não seria surpreendente, considerando-se o papel central que certos recursos desempenham nas teorias da EMN. No entanto, esse artigo de Barney representa apenas 3,2\% das citações, presente em apenas 7 artigos. Já as citações de Visão Baseada em Instituições e Global Value Chain - que poderiam ser relacionadas à classificação $\mathrm{AMB}$, mais atual segundo Buckley (2000), correspondem a apenas 5\% das citações.

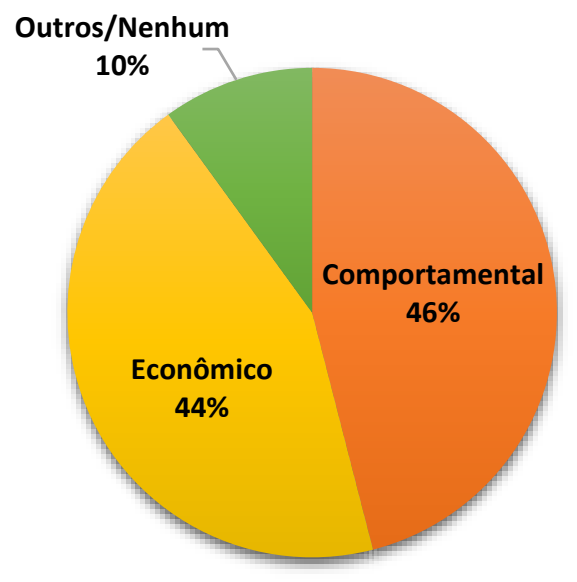

Figura 6: Internacionalização - grupos de teorias Fonte: Elaborado pelos autores

\subsection{Demografia de autoria}

Os números de artigos foram contados de acordo com os critérios descritos na Metodologia. A afiliação do autor a IES refere-se à mais atual, de acordo com currículo Lattes.

Tab. 7

Autoria

\begin{tabular}{ccc}
\hline Autores & Artigos & IES \\
\hline REZENDE S F L & 12 & PUC/MG \\
BORINI F M & 8 & ESPM \\
VERSIANI A & 8 & PUC/MG \\
BOEHE D M & 6 & Adelaide \\
FLEURY M T L & 5 & EAESP/FGV \\
OLIVEIRA JR M M & 5 & FEA/USP \\
RODRIGUES S B & 3 & FUMEC \\
SHENG H H & 3 & EAESP/FGV \\
\hline
\end{tabular}

Fonte: Elaborado pelos autores

Os 80 artigos analisados foram escritos por 127 autores diferentes, pertencentes a $51 \mathrm{IES}$. Os oito autores mais produtivos estão na tabela 6 , com o número de artigos em que aparecem na autoria. Os outros 119 produziram dois (11 autores) ou apenas um artigo (108 autores). Dos mais produtivos, apenas um é de IES pública, sendo quatro de IES privadas, dois de confessional e um encontra-se fora do Brasil. Das 51 IES que aparecem na autoria dos artigos, um grupo de seis se destaca com maior produção.

Tab. 8

Instituição de Ensino Superior (IES)

\begin{tabular}{lccc}
\hline IES & Artigos & IES & Freq $\%$ \\
\hline PUC MG & 26 & & \\
FEA USP & 20 & & \\
EAESP FGV & 16 & & \\
ESPM & 13 & & \\
UFRGS & 7 & & \\
UNISINOS & 7 & & \\
\hline Mais produtivas & & 6 & $12 \%$ \\
\hline 5 ou 6 artigos & & 3 & $6 \%$ \\
3 ou 4 artigos & & 11 & $22 \%$ \\
1 ou 2 artigos & & 31 & $61 \%$ \\
\hline Total & & 51 & \\
\hline
\end{tabular}

Fonte: Elaborado pelos autores

As mesmas IES da tabela 8 aparecem no trabalho de Forte, Rodrigues e Oliveira (2010), com exceção da ESPM, que inicia seu programa de pós-graduação em Gestão Internacional em 2010, e começa, só então, a aparecer nas listas de publicações.

As instituições às quais pertencem os autores foram classificadas como públicas, privadas e confessionais, de acordo com a definição constante nos artigos 19 e 20 da Lei de Diretrizes e bases da Educação Brasileira (BRASIL, 1996). O resultado encontra-se na Figura 7. A maior quantidade de artigos é originária das instituições públicas (44\%), seguidas das privadas (34\%), e das confessionais com apenas $14 \%$ das publicações, apesar de serem base de alguns dos autores mais prolíficos.

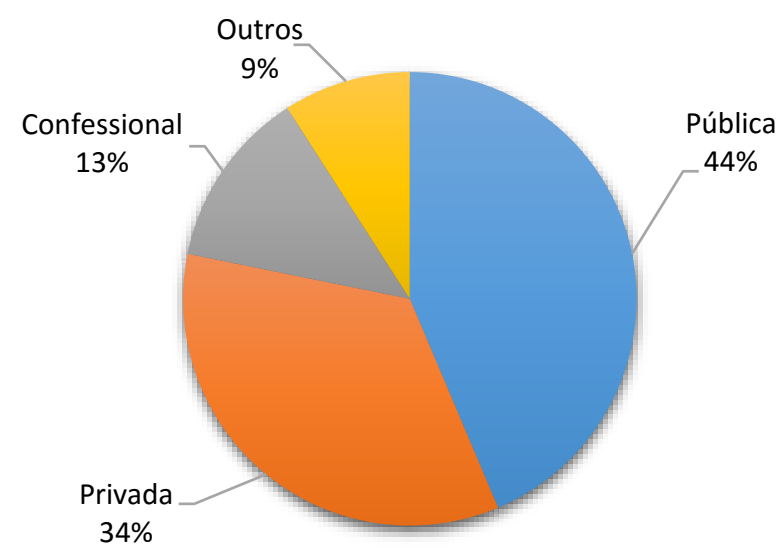

Figura 7: Tipo das IES dos autores Fonte: Elaborado pelos autores 
O estudo realizado por Forte, Rodrigues e Oliveira (2010) nos artigos apresentados nos eventos da ANPAD, também aponta para a presença significativamente maior de instituições públicas (39\%).

\section{Considerações finais}

A produção acadêmica sobre internacionalização no Brasil apresenta uma tabela de crescimento em relação ao início do séc. XXI, notadamente de 2006 a 2009, a partira de quando o número de artigos anuais praticamente se estabiliza. Esse crescimento pode ser atribuído ao aumento do número de periódicos (BAR), ao início da publicação de trabalhos sobre internacionalização em periódicos tradicionais (RAE/FGV), e ao surgimento de novos programas de pós-graduação em gestão internacional (ESPM). A produção é concentrada em poucos autores e poucas instituições, com grande número de autores que publicam apenas um artigo e não dão continuidade à publicação, o que pode ser considerado um indicador de uma área de estudos ainda não amadurecida (SPLITTER; ROSA; BORBA, 2012).

Em termos de conteúdo, algumas questões podem ser levantadas como conclusão do presente estudo.

Em primeiro lugar, a temática menos frequente é a análise do ambiente e estudos de globalização (18\% dos artigos), enquanto que nos artigos publicados no Journal of International Business Studies ela é responsável por 29\% (Amatucci, 2006a). Em 2002 Buckley (2002) já apontava que essa abordagem estaria para ser superada, e parece que ainda nem chegou a se desenvolver na produção nacional. Essa constatação pode mostrar, antes de uma possível deficiência da academia brasileira, uma realidade do país, pois essa agenda de pesquisa toca em assuntos como significados da globalização, competitividade e outras questões distantes do estágio das empresas locais, em uma economia fechada ao comércio internacional.

Em segundo lugar, a metodologia empregada difere de estudos sobre administração. Estudo internacional (SCANDURA, WILLIAMS, 2000) mostra apenas $4 \%$ de surveys nas pesquisas sobre temas gerais da administração, enquanto que no presente estudo chegaram a $21 \%$. Por outro lado, nos artigos do JIBS esse número chegou a 35\% (Amatucci, 2006a). Isso talvez indique que a pesquisa sobre internacionalização pode ter uma natureza intrinsicamente diferente da pesquisa geral sobre administração com relação a surveys.

Em terceiro lugar, o emprego de teorias no Brasil difere da academia internacional. Aqui são mais usadas as teorias "comportamentais" (46\%), enquanto que na academia internacional as "econômicas" predominam (46\%) (Amatucci, 2006a). Isso pode estar relacionado à maior frequência de estudos de caso na pesquisa brasileira (46\%). O estudo das teorias presentes na literatura avaliada é uma das contribuições desse estudo, pois não é comum esse tipo de abordagem quando se faz uma revisão bibliográfica de uma área da administração.

No entanto, é oportuno ressaltar limitações da presente pesquisa.

A primeira é que a amostra foi restrita em termos de número de periódicos, o que permite avaliar esse grupo - significativo em termos de qualidade, porém pequeno - mas não se pode traçar um panorama mais amplo e preciso da produção acadêmica brasileira em internacionalização de empresas. Para esse objetivo ser atingido, a pesquisa deveria ser ampliada para o estudo dos periódicos classificados pelo sistema Qualis como B1, B2, B3, bem como incluir, com diferente ponderação, os trabalhos apresentados em congressos.

A segunda limitação é a reduzida base de comparação, para se ter uma visão da pesquisa da área de internacionalização em comparação com outras áreas da administração e também com a pesquisa sobre internacionalização em outros contextos acadêmicos.

Também não foram contempladas as publicações de autores brasileiros em periódicos internacionais, o que poderia indicar a ampliação da abrangência da produção acadêmica brasileira, e a área de internacionalização seria a mais favorável a isso.

Essas limitações indicam, ao mesmo tempo, possíveis desdobramentos futuros da presente pesquisa.

\section{References}

- AlCAdipani, R.; DAVEl, E. Estudos críticos em administração: a produção científica brasileira nos anos 1990. RAE, v. 43, n. 4, p. 72-84, 2003. DOI: http://dx.doi.org/10.1590/S003475902003000400006 
- AMATUCCI, M. Temas e metodologias em negócios internacionais: um estudo longitudinal. Revista Gerenciais, São Paulo, v. 5, n. 2, p. 83-90, 2006(b).

Temas e raízes teóricas em negócios internacionais: um estudo longitudinal. In: SEGET. Simpósio de Excelência em Gestão e Tecnologia, 2006, Rezende - RJ. Anais... V SEGET. Rezende - RJ: AEDB-RJ, v. 1. p. 1-16, 2006(a).

- ANDERSSON, S. The Internationalization of the firm from an entrepreneurial perspective. International Studies of Management \& Organization, v. 30, n. 1, p. 63-92, Spring 2000.

- ANDERSSON, U.; FORSGREN, M.; HOLM, U. The strategic impact of external networks: subsidiary performance and competence development in the multinational corporation. Strategic Management Journal, v. 23, 979-996, 2002.

- ANDRADE, M. A. M.; JOAQUIM, N. F.; GOSLING, M. Metodologias de análise de imagem no marketing: um estudo bibliométrico dos anais do EnANPAD. In: V EMA - Encontro de Marketing da ANPAD, 2012, Rio de Janeiro. Anais... Rio de Janeiro: ANPAD, 2012.

- ARaújo, R. M.; AZeVedo, A. K.; VIeIRA, L. L.; NASCIMENTO, T. C. Periódicos em ação: um estudo exploratório-bibliométrico na área de Administração, Ciências Contábeis e Turismo. Perspectivas em Ciência da Informação, v. 19, n. 1, p. 90-114, 2014.

- ARKADER, R. A pesquisa científica em gerência de operações no Brasil. RAE, v. 43, n. 1, p. 70-80, 2003. DOI: $\quad$ http://dx.doi.org/10.1590/S003475902003000100008

- BARNEY, J.B. Firm resources and sustained competitive advantage. Journal of Management, v. 17, p. 99-120. 1991.

- Bertero, C. O. Pesquisa em internacionalização de empresas. (Palestra). In: III CONGRESSO DE ADMINISTRAÇÃO DA ESPM. São Paulo, 26 a 28 de julho de 2006. Anais... São Paulo: CAEPM, 2006 (CD ROM).

- Bertero, C. O.; VAsconcelos, F. C.; Binder, M. P. Estratégia empresarial: a produção científica brasileira entre 1991 e 2002. RAE, v. 43, n. 1, p. 78-62, 2003.

- BIRKINSHAW, J. Entrepreneurship in multinational corporations: the characteristics of subsidiary initiatives. Strategic Management Journal, v. 18, n. 3, p. 207-29, 1997.

- BIRKINSHAW, J; HOOD, N. Multinational subsidiary evolution: Capability and charter change in foreignowed subsidiaries companies. Academy of Management Review, v. 23, n.4, p.773-795, 1998.

- BORINI, F. M.; COELHO, F. P.; RIBEIRO, F. C. F.; PROENÇA, E. R. O Prisma da Internacionalização. In: VII SEMEAD: Seminários em Administração FEA/USP. São Paulo, 10 e 11 de agosto de 2004. Anais... São Paulo: FEA/USP, 2004.
- BRASIL Lei no 9.394, de 20 de dezembro de 1996. Lei de Diretrizes e Bases da Educação Nacional. Estabelece as diretrizes e bases da educação nacional. 1996.

- BUCKLEY, P. J. Is the international business research agenda running out of steam? Journal of International Business Studies, v. 33, n. 2, p. 365-373, 2002.

- BUCKLEY, P.; CASSON, M. The future of the multinational enterprise. London: The Macmillan Press, 1976.

- CAldas, M. P.; TINOCO, T. Pesquisa em gestão de recursos humanos nos anos 1990: um estudo bibliométrico. RAE, v. 44, n. 3, p. 100-114, 2004.

- CAMPOS, M. Conceitos atuais em bibliometria. Arquivos Brasileiros Oftalmologia, v. 66, n. p. 1-22, 2003.

- CAPES - $\quad$ WebQualis

http://qualis.capes.gov.br/webqualis/publico/pesquisa PublicaClassificacao.seam?conversationPropagation $=b$ egin [Acesso em 31/10/2014]

- $\quad$ CARdoso, R. L.; MENDONÇA NETO, O. R.; RICCIO, E. L.; SAKATA, M. C. G. Pesquisa científica em contabilidade entre 1990 e 2003. RAE, v. 45, n. 2, p. 34-45, 2005.

- CARNeIRO, J.; DIB, L. A. Avaliação comparativa do escopo descritivo e explanatório dos principais modelos de internacionalização de empresas. INTERNEXT Revista Eletrônica de Negócios Internacionais da ESPM, v. 2, n. 1, p. 1-25, 2007.

- CAVES, R. International Corporations: the industrial economics of foreign investment, Economica, v. 38 february, 1971, p. 1-27.

- COASE, R. The nature of the firm. Economica, v. 4, n. 16, p.386-405, 1937.

- DUNNING, J. H. Multinational Enterprises and the Global Economy. Workhingan: Addison-Wesley, 1993.

- $\quad$ Some antecedents of internalization theory. Journal of International Business Studies, 34. p.108-15, 2003.

- . The eclectic paradigm of international production: a restatement and some possible extensions. Journal of International Business Studies, v. 19, n. 1, p.1-31, 1988.

The eclectic paradigm of internationa production: a personal perspective. In: PITELIS, C.N.; SUGDEN, R. (ed.) The nature of the transnational. London: Routledge, 1991. Chapter 5, p.117-36.

Toward an eclectic theory of international production: some empirical tests. Journal of International Business Studies, v. 2, n. 3, p. 9-31, 1980.

Trade, location of economic activity, and the multinational enterprise: a search for an eclectic approach. In: OHLIN, B. et al (ed.) The international allocation of economic activity. London: Macmillan, 1977. 
- FAVORETO, R. L.; AMÂNCIO-VIEIRA, S. F.; SHIMAdA, A. T. A produção intelectual em RBV: uma incursão bibliométrica nos principais periódicos nacionais. REBRAE - Revista Brasileira de Estratégia, v.7, n.1, p. 3755,2014

- FORSGREN, M. Foreign acquisitions: internationalization or network dependency? Advances in International Marketing. Greenwich: JAI Press, v. 3, p. 141-59, 1989.

- $\quad$ fORTE, S. H. A. C.; ROdRIGUES, D. P.; OliVEIRA, O. V. Diagnóstico das Pesquisas em Gestão Internacional no Brasil. Estratégia e Negócios. v. 3, n. 1, 2010.

- GEREFFI, G., J.; HUMPhREY, J.; STURGEON, T. The Governance of Global Value Chains. Review of International Political Economy, v. 12 n. 1, p. 78-104, 2005.

- HEMAIS, C. A.; HILAL, A. Teorias, paradigmas e tendências em negócios internacionais: de Hymer ao empreendedorismo. In: HEMAIS, C. A. (Org.): O desafio dos mercados externos: teoria e prática na internacionalização da firma. Rio de Janeiro: Mauad, 2004; v. I, p. 17-39.

- HOPPEN, N.; MEIRELLES, F. S. Sistemas de informação: um panorama da pesquisa científica entre 1990 e 2003. RAE, v. 45, n. 1, p. 24-35, 2005.

- HYMER, S. The international operations of national firms: a study of direct foreign investment. Doctorate thesis. Cambridge, MA: The MIT Press, 1960/1976.

- jabBour, C. J. C.; SANTOS F. C. A.; BARBIERI, J. C. Gestão ambiental empresarial: um levantamento da produção científica brasileira divulgada em periódicos da área de administração entre 1996 e 2005. RAC, v. 12, n. 3, p. 689-715, 2008.

- JOHANSON, J.; MATTSON, L. International marketing and internationalization processes - a network approach. In: PALIWODA, S.; TURNBULL, P.W. (ed.) Research in international marketing. London: Croom Helm, 1986.

- JOHANSON, J.; VAHLNE, J. Business relationship learning and commitment in the internationalization process. Journal of International Entrepreneurship, v. 1, p. 83-101, 2003

The internationalization process of the firm: a model of knowledge and increasing foreign market commitment. Journal of International Business Studies, v. 8, n. 1, p. 23-32, 1977.

The mechanism of internationalisation. International Marketing Review, v. 7, n. 4, p.11-24, 1990.

- JOHANSON, J.; WIEDERSHEIM-PAUL, F. The internationalization of the firm: four Swedish cases. Journal of Management Studies, v. 12, n. 3, p. 305-22, 1975.

- KRUGMAN, P. R. Geography and Trade, Cambridge: MIT. 1991.
- LeAl, R. P. C.; OliveirA, J.; SOluRI, A. F. Perfil da pesquisa em finanças no Brasil. RAE, v. 43, n. 1, p. 91 104, 2003. DOI: http://dx.doi.org/10.1590/S003475902003000100010

- MACHADO-DA-SILVA, C. L.; CUNHA, V. C.; AMBONI, N. Organizações: o estado da arte da produção acadêmica no Brasil. Anais... Encontro Nacional da Associação Nacional de Pós-Graduação e Pesquisa em Administração, Florianópolis, SC, Brasil, 14. 1990.

- MARIOTTO, F. L. Estratégia Internacional da Empresa. São Paulo: Thomson Learning, 2007. Coleção Debates em Administração. 131 p.

- Martignago, G.; Alperstedt, G. D.; CÁRIO, S. A. F. Pesquisa em gestão internacional no Brasil entre 2001 e 2010: um estudo a partir dos anais do EnANPAD. Estratégia \& Negócios, v. 6, n. 2, p. p. 215-243, 2013. DOI: http://dx.doi.org/10.1590/S141565552010000200006

- MCGRATH, J. E. Dillemmatics: the study of research choices and dilemmas. In: MCGRATH, J. E.; MARTIN, J.; KULKA, R. A. (Ed) Judgement calls in research. Beverly Hilss: Sage Publications, p. 69-102, 1982.

- MENDONÇA NETO, O. R.; CARDOSO, R. L.; RICCIO, E. L.; SAKATA, M. C. G. Dez anos de pesquisa contábil no Brasil: análise dos trabalhos apresentados nos EnANPADs de 1996 a 2005. RAE, v. 49, n. 1, p. 62-73, 2009.

- ogasavara, M. H.; MASIERO, G. Reviewing recent developments on research of internationalization of Brazilian firms. XXXIII EnANPAD. São Paulo, 19 a 23 de setembro de 2009. Anais... Rio e Janeiro: ANPAD, 2009, CD-ROM.

- PACHECO, R. S. Administração pública nas revistas especializadas - Brasil, 1995-2002. RAE, v. 43, n. 4, p. 63 -71, 2003. DOI: http://dx.doi.org/10.1590/S003475902003000400005

- PENG, M. The global strategy of emerging multinationals from China. Global Strategy Journal, v. 2, n. 2, p. 97-107. 2012. DOI: DOI: 10.1002/gsj.1030

- $\quad$ PENG, M. W.; SUN, S. L.; PINKHAM, B.; CHEN, H. The institution-based view as a third leg for a strategy tripod. Academy of Management Perspectives, v. 23, n. 4, p. 63-81. 2009

- $\quad$ PENG, M.W; WANG, D.Y.L.; JIANG, L. An institutionbased view of international business strategy: a focus on emerging economies. Journal of International Business Studies, v. 39, p. 920-936. 2008.

- PeReira, V. R.; CARVAlHO, M. M.; ROtONDARO, R. G. Um estudo bibliométrico sobre a evolução da pesquisa da qualidade em serviço. Produção. v. 23, n. 2, p. 312328, 2013. DOI: http://dx.doi.org/10.1590/\$010365132012005000053

- PNPG 2011-2020 Relatório Final Comissão Especial de Acompanhamento do PNPG 2011-2020 e Elaboração da Agenda Nacional de Pesquisa. 2013. Disponível em <http://www.capes.gov.br/images/stories/download/ 
PNPG-Relatorio-Final-11-12-2013.pdf>. Acesso em 14/07/2015.

- PORTER, M. E. The Competitive Advantage of Nations Free Press, New York, 1990.

- RUgMan, A. Inside the Multinationals. Columbia University Press, New York, NY, 1981.

- SCANDURA, T. A.; WILLIAMS, E. A. Research methodology in management: current practices, trends, and implications for future research. The Academy of Management Journal, v. 43, n. 6, p. 12481264, 2000.

- SIQUeIRA, M. O tema recursos humanos nas reuniões da ANPAD: trajetórias e perspectivas. XII EnANPAD. Natal, RN, 26 a 28 de setembro de 1988. Anais... Belo Horizonte: ANPAD, 1988.

- $\quad$ SPLITTER, K.; ROSA, C. A.; BORBA, J. A. Uma Análise das Características dos Trabalhos "Ditos" Bibliométricos Publicados no EnANPAD entre 2000 e 2011. In: XXXVI EnANPAD, Rio de Janeiro, 2012. Anais... XXXVI EnANPAD.

- TEECE, D. The multinational enterprise: market failure and market power considerations. Sloan Management Review, n. 22, p. 3-17, 1981.
- TEECE, D. Transaction cost economics and the multinational enterprise. Journal of Economic Behaviour and Organization, n. 7, p. 21-45, 1986.

- THEÓPHILO, C. R.; IUDíCIBUS, S. Uma análise críticoepistemológica da produção científica em Contabilidade no Brasil. Contabilidade, Gestão e Governança, v. 8, n. 2, p. 147-175, 2005.

- TONelli, M. J.; CALDAS, M. P.; LACOMBE, B. M. B.; TINOCO, T. Produção acadêmica em recursos humanos no Brasil: 1991-2000. RAE, v. 43, n. 1, p. 105-122, 2003

- VERNON, R. International investment and international trade in the product cycle. Quarterly Journal of Economics, v. 80, p. 190-207, 1966.

- VIEIRA, F. G. D. - Narciso sem espelho: a publicação brasileira de marketing. RAE, v. 43, n. 1, p. 81-90, 2003.

- WILLIAMSON, O. Markets and hierarchies: analysis and antitrust implications. New York: Free Press, 1975. Transaction-cost economics: the governance of contractual relations. The Journal of Law and Economics, v. 22, p. 233-61, Oct. 1979.

\section{SOBRE OS AUTORESS}

- Sergio Garrido Moraes é Doutorando no Programa de Mestrado e Doutorado em Gestão Internacional - PMDGI e Docente nos cursos de administração e jornalismo da Escola Superior de Propaganda e Marketing -ESPM, São Paulo/SP, Brasil.Email: smoraes@espm.br

- Vivian lara Strehlau é Doutora em Administração de Empresas pela Fundação Getúlio Vargas (EAESP/FGV) e Docente no Programa de Mestrado e Doutorado em Gestão Internacional da Escola Superior de Propaganda e Marketing - ESPM, São Paulo/SP, Brasil. Email: vstrehlau@espm.br

- Frederico Araújo Turolla é Doutor em Administração de Empresas pela Fundação Getúlio Vargas (EAESP/FGV) e Docente no Programa de Mestrado e Doutorado em Gestão Internacional da Escola Superior de Propaganda e Marketing - ESPM, São Paulo/SP, Brasil. Email:fturolla@espm.br 


\title{
Academic production of Brazilian authors on internationalization: Balance of the publications in Brazil in the $21^{\text {st }}$ century
}

Sergio Garrido Moraes, Vivian Iara Strehlau and Frederico Araújo Turolla

Escola Superior de Propaganda e Marketing - ESPM, São Paulo/SP, Brasil

\begin{tabular}{l}
\hline ARTICLE DETAILS \\
\hline Article history: \\
Received 31 October 2014 \\
Accepted 27 July 2015 \\
Available online in 30 August 2015 \\
Double Blind Review System \\
Scientific Editors \\
Gabriel Vouga Chueke \\
Marcos Amatucci \\
\hline Keywords: \\
Internationalization \\
academic production \\
balance of the literature
\end{tabular}

\begin{abstract}
This paper presents an analysis of Brazilian scientific research on Internationalization of Companies, through a review of all the articles published in this area in journals classified by CAPES as Qualis A, from 2001 to 2014, and its comparison with certain indicators from other studies on academic research in Brazil and abroad, on internationalization as well as management themes in general. The analysis focuses on the themes addressed, the methodology used, the internationalization theories discussed and authorship demographics. It was found that possible differences may exist in Brazilian research on internationalization compared to how it is conducted in other countries in relation to themes, methodology and theories. In terms of authorship demographics, it can be seen that the contribution of public and private higher education institutions is in line with other areas. Lastly, possibilities are suggested for expansion of the study to better reflect Brazilian scientific production in Internationalization of Companies.
\end{abstract}

(C) 2015 Internext | ESPM. All rights reserved! 